\title{
Compact Solvmanifolds with a Closed $\mathrm{G}_{2}$-Structure
}

\section{Marisa Fernández, Jonatan Sanchez, Jose A. Santisteban}

Faculty of Science and Technology, Department of Mathematics, University of the Basque Country (UPV/EHU), Bilbao, Spain

Email: marisa.fernandez@ehu.es, jonatan.sanchez@ehu.eus, joseba.santisteban@ehu.eus

How to cite this paper: Fernández, M., Sanchez, J. and Santisteban, J.A. (2018) Compact Solvmanifolds with a Closed $\mathrm{G}_{2}$-Structure. Advances in Pure Mathematics, 8, 813-829. https://doi.org/10.4236/apm.2018.811050

Received: October 15, 2018

Accepted: November 24, 2018

Published: November 27, 2018

Copyright (C) 2018 by authors and Scientific Research Publishing Inc. This work is licensed under the Creative Commons Attribution International License (CC BY 4.0). http://creativecommons.org/licenses/by/4.0/

\begin{abstract}
We consider a parametrized family of compact $\mathrm{G}_{2}$-calibrated solvmanifolds, and construct associative (so volume-minimizing submanifolds) 3-tori with respect to the closed $\mathrm{G}_{2}$-structure. We also study the Laplacian flow of this closed $\mathrm{G}_{2}$ form on the solvable Lie group underlying to each of these solvmanifolds, and show long time existence of the solution.
\end{abstract}

\section{Keywords}

$\mathrm{G}_{2}$-Structure, Laplacian Flow, Associative 3-Folds

\section{Introduction}

A $\mathrm{G}_{2}$-structure on a seven-dimensional manifold $M$ is defined by a positive 3-form $\varphi$ (the $\mathrm{G}_{2}$ form) on $M$, which induces a Riemannian metric $g_{\varphi}$ and a volume form $d V_{\varphi}$ on $M$ such that

$$
g_{\varphi}(X, Y) d V_{\varphi}=\frac{1}{6} l_{X} \varphi \wedge l_{Y} \varphi \wedge \varphi,
$$

for any vector fields $X, Y$ on $M$. If the 3 -form $\varphi$ is covariantly constant with respect to the Levi-Civita connection of the metric $g_{\varphi}$ or, equivalently, the 3 -form $\varphi$ is closed and coclosed [1], then the holonomy group of $g_{\varphi}$ is a subgroup of the exceptional Lie group $\mathrm{G}_{2}$, and the metric $g_{\varphi}$ is Ricci-flat. When this happens, the $\mathrm{G}_{2}$-structure is said to be torsion-free [2]. The first compact examples of Riemannian manifolds with holonomy $\mathrm{G}_{2}$ were constructed first by Joyce [3], and then by Kovalev [4]. Recently, other examples of compact manifolds with holonomy $\mathrm{G}_{2}$ were obtained in [5] [6].

There are many different $\mathrm{G}_{2}$-structures attending to the behavior of the exterior derivative of the $G_{2}$ form [1] [7]. In the following, we will focus our 
attention on $\mathrm{G}_{2}$-structures where the 3 -form $\varphi$ is closed. In this case, the $\mathrm{G}_{2}$-structure is said to be closed (or calibrated). The first example of a compact $\mathrm{G}_{2}$-calibrated manifold, which does not admit any torsion-free $\mathrm{G}_{2}$-structure, was obtained in [8]. This example is a compact nilmanifold, that is a compact quotient of a simply connected nilpotent Lie group by a lattice, endowed with an invariant calibrated $\mathrm{G}_{2}$-structure. In [9], Conti and the first author classified the 7-dimensional compact nilmanifolds admitting a left invariant closed $\mathrm{G}_{2}$-structure. More examples were given in [10] [11] [12] [13].

Calibrated geometry was introduced by Harvey and Lawson in [14] and it concerns to a special type of minimal submanifolds of a Riemannian manifold, which are defined by a closed form (the calibration) on the manifold. Such submanifoldds are called calibrated submanifolds (see Section 5 for details). Every compact calibrated submanifold is volume-minimizing in its homology class ([15] Proposition 3.7.2).

In addition to compact Kähler manifolds and compact 7-manifolds with a torsion-free $\mathrm{G}_{2}$-structure, 7-manifolds with a closed $\mathrm{G}_{2}$-structure are also calibrated manifolds. In fact, if $M$ is a 7-manifold with a closed $\mathrm{G}_{2}$-structure $\varphi$, then $\varphi$ is a calibration [14]. The 3-dimensional orientable submanifolds $Y \subset M$ calibrated by the $\mathrm{G}_{2}$ form $\varphi$, that is, those 3-dimensional submanifolds $Y \subset M$ such that $\varphi$ restricted to $Y$ is a volume form for $Y$, are called associative 3 -folds of $(M, \varphi)$.

In this paper, we consider a parametrized family of 7-dimensional compact solvmanifolds $M^{7}(k)$ with an invariant closed $\mathrm{G}_{2}$-structure $\varphi_{k}$, which is not coclosed, where $k$ is a real number such that $e^{k}+e^{-k}$ is an integer number different from 2. We show that $M^{7}(k)$ is formal (Proposition 4.1) and its first Betti number $b_{1}\left(M^{7}(k)\right)=3$. Moreover, we construct associative calibrated (so volume-minimizing) 3-tori in $M^{7}(k)$ with respect to the closed $\mathrm{G}_{2}$ form $\varphi_{k}$ (Proposition 5.3).

By [16] [17], a closed $\mathrm{G}_{2}$-structure on a compact manifold cannot induce an Einstein metric, unless the induced metric has holonomy contained in $\mathrm{G}_{2}$. It is still an open problem to see if the same property holds on noncompact manifolds. For the homogeneous case, a negative answer has been recently given in [18]. Indeed, in [18] it is proved that if a solvable Lie algebra has a closed $\mathrm{G}_{2}$-structure then the induced inner product is Einstein if and only if it is flat.

Natural generalizations of Einstein metrics are given by Ricci solitons, which have been introduced by Hamilton in [19]. All known examples of nontrivial homogeneous Ricci solitons are solsolitons. They are right invariant (or left invariant) metrics on simply connected solvable Lie groups, whose Ricci curvature tensor satisfies the condition

$$
\operatorname{Ric}(g)=\lambda I+D
$$

for some $\lambda \in \mathbb{R}$ and some derivation $D$ of the corresponding Lie algebra, where $I$ is the identity map.

A natural question is thus to see if a closed $\mathrm{G}_{2}$-structure on a noncompact 
manifold induces a (non-Einstein) Ricci soliton metric. For the metric determined by the invariant closed $\mathrm{G}_{2}$ form $\varphi_{k}$ on $M^{7}(k)$ mentioned before, we show that if $H(k)$ is the simply connected solvable (non-nilpotent) Lie group underlying to $M^{7}(k)$, then $\varphi_{k}$ induces a solsoliton on $H(k)$ (see Proposition 4.2).

The other motivation of this paper comes from the Laplacian flow on 7-manifolds admitting closed $\mathrm{G}_{2}$-structures. Let $M$ be a 7-dimensional manifold with a closed $\mathrm{G}_{2}$-structure $\varphi$. The Laplacian flow on $M$ starting from $\varphi$ is given by

$$
\left\{\begin{array}{l}
\frac{\partial}{\partial t} \varphi(t)=\Delta_{t} \varphi(t), \\
d \varphi(t)=0, \\
\varphi(0)=\varphi,
\end{array}\right.
$$

where $\varphi(t)$ is a closed $\mathrm{G}_{2}$ form on $M$ and $\Delta_{t}=d d^{*}+d^{*} d$ is the Hodge Laplacian operator associated with the metric $g_{\varphi(t)}$ induced by the 3 -form $\varphi(t)$. This geometric flow was introduced by Bryant in [16] as a tool to find torsion-free $\mathrm{G}_{2}$-structures on compact manifolds. Short-time existence and uniqueness of the solution, in the case of compact manifolds, were proved in [20]. Properties of this flow were proved in [21] [22] [23].

The first noncompact examples with long-time existence of the solution were obtained on seven-dimensional nilpotent Lie groups in [24], but in those examples the Riemannian curvature tends to 0 as $t$ goes to infinity. Further solutions on solvable Lie groups were described in [25] [26] [27] [28]. Moreover, a cohomogeneity one solution converging to a torsion-free $G_{2}$-structure on the 7-torus was worked out in [29].

In Section 6, we consider the solvable (non-nilpotent) Lie group $H(k)$ underlying to the compact solvmanifold $M^{7}(k)$, and we show that the Laplacian flow of $\varphi_{k}$ on $H(k)$ exists for all time. In fact, in Theorem 6.2, we explicitly determine the solution $\varphi_{k}(t)$ for the flow of $\varphi_{k}$ on $H(k)$, and we prove that it is defined on a time interval of the form $(T, \infty)$, where $T<0$ is a real number. (This solution was previously given in [25] from a family of symplectic half-flat structures on a 6-dimensional ideal of the Lie algebra $\mathfrak{h}(k)$ of $H(k)$.) We also show that the Ricci endomorphism Ric $\left(g_{k}(t)\right)$ of the underlying metric $g_{k}(t)$ of $\varphi_{k}(t)$ is independent of the time $t$, and so the solution $\varphi_{k}(t)$ does not converge to a torsion-free $\mathrm{G}_{2}$-structure as $t$ goes to infinity.

\section{Closed $\mathrm{G}_{2}$-Structures}

In this section we collect some basic facts and definitions concerning $G_{2}$ forms on smooth manifolds (see [1] [2] [7] [14] [15] [16] [30] [31] [32] [33] for details).

Let us consider the space $\mathbb{O}$ of the Cayley numbers, which is a non-associative algebra over $\mathbb{R}$ of dimension 8 . Thus, we can identify $\mathbb{R}^{7}$ 
with the subspace of $\mathbb{O}$ consisting of pure imaginary Cayley numbers. Then, the product on $\mathbb{O}$ defines on $\mathbb{R}^{7}$ the 3 -form given by

$$
e^{127}+e^{347}+e^{567}+e^{135}-e^{236}-e^{146}-e^{245}
$$

(see [1] [32] [33] [34] for details), where $\left\{e^{1}, \cdots, e^{7}\right\}$ is the standard basis of $\left(\mathbb{R}^{7}\right)^{*}$. Here, $e^{127}$ stands for $e^{1} \wedge e^{2} \wedge e^{7}$, and so on. The group $\mathrm{G}_{2}$ is the stabilizer of (2) under the standard action of $\operatorname{GL}(7, \mathbb{R})$ on $\Lambda^{3}\left(\mathbb{R}^{7}\right)^{*} \cdot \mathrm{G}_{2}$ is one of the exceptional Lie groups, and it is a compact, connected, simply connected simple Lie subgroup of $\mathrm{SO}(7)$ of dimension 14.

$\mathrm{A} \mathrm{G}_{2}-$ structure on a 7-dimensional manifold $M$ is a reduction of the structure group of its frame bundle from $\operatorname{GL}(7, \mathbb{R})$ to the exceptional Lie group $G_{2}$, which can actually be viewed naturally as a subgroup of $\mathrm{SO}(7)$. Thus, a $\mathrm{G}_{2}$-structure determines a Riemannian metric and an orientation on $M$. In fact, one can prove that the existence of $\mathrm{a}_{2}$-structure is equivalent to the existence of a global differential 3-form $\varphi$ (the $\mathrm{G}_{2}$ form) on $M$, which can be locally written as (2) with respect to some (local) basis $\left\{e^{1}, \cdots, e^{7}\right\}$ of the (local) 1 -forms on $M$. Such a 3 -form $\varphi$ was introduced by Bonan in [35], and it induces a Riemannian metric $g_{\varphi}$ and a volume form $d V_{\varphi}$ on $M$ satisfying (1). We say that the manifold $M$ has a closed (or calibrated) $\mathrm{G}_{2}$-structure if there is a $\mathrm{G}_{2}$-structure $\varphi$ on $M$ such that $\varphi$ is closed, that is $d \varphi=0$, and so $\varphi$ defines a calibration [14].

Now, let $G$ be a 7-dimensional simply connected nilpotent Lie group with Lie algebra $\mathfrak{g}$. Then, a $\mathrm{G}_{2}$-structure on $G$ is left invariant if and only if the corresponding 3-form $\varphi$ is left invariant. Thus, a left invariant $\mathrm{G}_{2}$-structure on $G$ corresponds to an element $\varphi$ of $\Lambda^{3}\left(\mathfrak{g}^{*}\right)$ that can be written as (2), that is,

$$
\varphi=e^{127}+e^{347}+e^{567}+e^{135}-e^{146}-e^{236}-e^{245},
$$

with respect to some orthonormal coframe $\left\{e^{1}, \cdots, e^{7}\right\}$ of the dual space $\mathfrak{g}^{*}$. We say that a $\mathrm{G}_{2}$-structure on $\mathfrak{g}$ is calibrated if $\varphi$ is closed, i.e.

$$
d \varphi=0,
$$

where $d$ denotes the Chevalley-Eilenberg differential on $\mathfrak{g}^{*}$. If $\Gamma$ is a discrete subgroup of $G, a_{2}$-structure on $\mathfrak{g}$ induces a $\mathrm{G}_{2}$-structure on the quotient $\Gamma \backslash G$. In particular, if $\mathfrak{g}$ is solvable and $\Gamma$ is a discrete subgroup of $G$ such that the quotient $\Gamma \backslash G$ is compact, then a $\mathrm{G}_{2}$-structure on $\mathfrak{g}$ determines a $\mathrm{G}_{2}$-structure on the compact manifold $\Gamma \backslash G$, which is called a compact solvmanifold; and if $\mathfrak{g}$ has a calibrated $\mathrm{G}_{2}$-structure, the $\mathrm{G}_{2}$-structure on $\Gamma \backslash G$ is also calibrated.

\section{Formal Manifolds}

First, we need some definitions and results about minimal models. Let $(A, d)$ be a differential algebra, that is, $A$ is a graded commutative algebra over the real numbers, with a differential $d$ which is a derivation, that is, $d(a \cdot b)=(d a) \cdot b+(-1)^{\operatorname{deg}(a)} a \cdot(d b)$, where $\operatorname{deg}(a)$ is the degree of $a$. 
A differential algebra $(A, d)$ is said to be minimal if it satisfies the following two conditions:

1) $A$ is free as an algebra, that is, $A$ is the free algebra $V$ over a graded vector space $V=\oplus V_{i}$,

2) there exists a collection of generators $\left\{a_{\tau}, \tau \in I\right\}$, for some well-ordered index set $I$, such that $\operatorname{deg}\left(a_{\mu}\right) \leq \operatorname{deg}\left(a_{\tau}\right)$ if $\mu<\tau$ and each $d a_{\tau}$ is expressed in terms of preceding $a_{\mu}(\mu<\tau)$. This implies that $d a_{\tau}$ does not have a linear part, that is, it lives in $\Lambda V^{>0} \cdot \Lambda V^{>0} \subset V$.

Morphisms between differential algebras are required to be degree-preserving algebra maps which commute with the differentials. Given a differential algebra $(A, d)$, we denote by $H^{*}(A)$ its cohomology. We say that $A$ is connected if $H^{0}(A)=\mathbb{R}$, and $A$ is one-connected if, in addition, $H^{1}(A)=0$.

We will say that $(\mathcal{M}, d)$ is a minimal model of the differential algebra $(A, d)$ if $(\mathcal{M}, d)$ is minimal and there exists a morphism of differential graded algebras $\rho:(\mathcal{M}, d) \rightarrow(A, d)$ inducing an isomorphism

$\rho^{*}: H^{*}(\mathcal{M}) \rightarrow H^{*}(A)$ on cohomology. Halperin [36] proved that any connected differential algebra $(A, d)$ has a minimal model unique up to isomorphism.

A minimal model $(\mathcal{M}, d)$ is said to be formal if there is a morphism of differential algebras $\Psi:(\mathcal{M}, d) \rightarrow\left(H^{*}(\mathcal{M}), d=0\right)$ that induces the identity on cohomology. The formality of a minimal model can be distinguished as follows.

Theorem 3.1 [37] $A$ minimal model $(\mathcal{M}, d)$ is formal if and only if $\mathcal{M}=\Lambda V$ and the space $V$ decomposes as a direct sum $V=C \oplus N$ with $d(C)=0, d$ is injective on $N$ and such that every closed element in the ideal $I(N)$ generated by $N$ in $\Lambda V$ is exact.

A minimal model of a connected differentiable manifold $M$ is a minimal model $(\Lambda V, d)$ for the de Rham complex $\left(\Omega^{*} M, d\right)$ of differential forms on $M$. If $M$ is a simply connected manifold, the dual of the real homotopy vector space $\pi_{i}(M) \otimes \mathbb{R}$ is isomorphic to $V^{i}$ for any $i$. (For details see, for example, [37] [38].)

Definition 3.2 We will say that a differentiable manifold $M$ is formal if its minimal model is formal or, equivalently, the differential algebras $\left(\Omega^{*} M, d\right)$ and $\left(H^{*}(M), d=0\right)$ have the same minimal model.

Many examples of formal manifolds are known: spheres, projective spaces, compact Lie groups, symmetric spaces, flag manifolds, and all compact Kähler manifolds [37].

We will also use the following property

Lemma 3.3 Let $M_{1}$ and $M_{2}$ be differentiable manifolds. Then, the product manifold $M=M_{1} \times M_{2}$ is formal if and only if $M_{1}$ and $M_{2}$ are formal.

In [39], the condition of formal manifold is weaken to s-formal manifold as follows.

Definition 3.4 Let $(\mathcal{M}, d)$ be a minimal model of a differentiable manifold $M$. We say that $(\mathcal{M}, d)$ is $s$-formal, or $M$ is an $s$-formal manifold $(s \geq 0)$ if 
$\mathcal{M}=\Lambda V$ such that for each $i \leq s$, the space $V^{i}$ of generators of degree $i$ decomposes as a direct sum $V^{i}=C^{i} \oplus N^{i}$, where the spaces $C^{i}$ and $N^{i}$ satisfy the three following conditions.

1) $d\left(C^{i}\right)=0$,

2) the differential map $d: N^{i} \rightarrow V$ is injective,

3) any closed element in the ideal $I_{s}=I_{s}\left(\bigoplus_{i \leq s} N^{i}\right)$, generated by $\bigoplus_{i \leq s} N^{i}$ in $\Lambda\left(\bigoplus_{i \leq s} V^{i}\right)$, is exact in $\Lambda V$.

The relation between the formality and the s-formality for a manifold is given in the following theorem.

Theorem 3.5 Let $M$ be a connected and orientable compact differentiable manifold of dimension $2 n$ or $(2 n-1)$. Then $M$ is formal if and only if it is $(n-1)$-formal.

\section{The Compact Solvmanifolds $M^{7}(k)$}

Let $G(k)$ be the simply connected and solvable Lie group of dimension 5 consisting of matrices of the form

$$
a=\left(\begin{array}{cccccc}
e^{k x_{5}} & 0 & 0 & 0 & 0 & x_{1} \\
0 & e^{-k x_{5}} & 0 & 0 & 0 & x_{2} \\
0 & 0 & e^{k x_{5}} & 0 & 0 & x_{3} \\
0 & 0 & 0 & e^{-k x_{5}} & 0 & x_{4} \\
0 & 0 & 0 & 0 & 1 & x_{5} \\
0 & 0 & 0 & 0 & 0 & 1
\end{array}\right),
$$

where $x_{i} \in \mathbb{R}$, for $1 \leq i \leq 5$, and $k$ is a real number such that $e^{k}+e^{-k}$ is an integer number different from 2. Then a global system of coordinates $\left\{x_{i}, 1 \leq i \leq 5\right\}$ for $G(k)$ is defined by $x_{i}(a)=x_{i}$, and a standard calculation shows that a basis for the right invariant 1-forms on $G(k)$ consists of

$$
\begin{aligned}
& e^{1}=d x_{1}-k x_{1} d x_{5}, \quad e^{2}=d x_{2}+k x_{2} d x_{5}, \\
& e^{3}=d x_{3}-k x_{3} d x_{5}, \quad e^{4}=d x_{4}+k x_{4} d x_{5}, \\
& e^{5}=d x_{5} .
\end{aligned}
$$

We notice that the Lie group $G(k)$ may be described as a semidirect product $G(k)=\mathbb{R} \ltimes_{\rho_{k}} \mathbb{R}^{4}$, where $\mathbb{R}$ acts on $\mathbb{R}^{4}$ via the linear transformation $\rho_{k}(t)$ of $\mathbb{R}^{4}$ given by the matrix

$$
\rho_{k}(t)=\left(\begin{array}{cccc}
e^{k t} & 0 & 0 & 0 \\
0 & e^{-k t} & 0 & 0 \\
0 & 0 & e^{k t} & 0 \\
0 & 0 & 0 & e^{-k t}
\end{array}\right) .
$$

Thus the operation on the group $G(k)$ is given by

$$
\boldsymbol{x} \cdot \boldsymbol{a}=\left(x_{1}+a_{1} e^{k x_{5}}, x_{2}+a_{2} e^{-k x_{5}}, x_{3}+a_{3} e^{k x_{5}}, x_{4}+a_{4} e^{-k x_{5}}, x_{5}+a_{5}\right),
$$

where $\boldsymbol{a}=\left(a_{1}, \cdots, a_{5}\right)$ and similarly for $\boldsymbol{x}$. Therefore $G(k)=\mathbb{R} \ltimes_{\rho_{k}} \mathbb{R}^{4}$, where 
$\mathbb{R}$ is a connected abelian subgroup, and $\mathbb{R}^{4}$ is the nilpotent commutator subgroup.

Now we show that there exists a discrete subgroup $\Gamma(k)$ of $G(k)$ such that the quotient space $G(k) / \Gamma(k)$ is compact. To construct $\Gamma(k)$ it suffices to find some real number $t_{0}$ such that the matrix defining $\rho_{k}\left(t_{0}\right)$ is conjugate to an element $A$ of the special linear group $\operatorname{SL}(4, \mathbb{Z})$ with distinct real eigenvalues $\lambda$ and $\lambda^{-1}$. Indeed, we could then find a lattice $\Gamma_{0}$ in $\mathbb{R}^{4}$ which is invariant under $\rho_{k}\left(t_{0}\right)$, and take $\Gamma(k)=\left(t_{0} \mathbb{Z}\right) \ltimes_{\rho_{k}} \Gamma_{0}$. To this end, we choose the matrix $A \in \mathrm{SL}(4, \mathbb{Z})$ given by

$$
A=\left(\begin{array}{llll}
2 & 1 & 0 & 0 \\
1 & 1 & 0 & 0 \\
0 & 0 & 2 & 1 \\
0 & 0 & 1 & 1
\end{array}\right),
$$

with double eigenvalues $\frac{3+\sqrt{5}}{2}$ and $\frac{3-\sqrt{5}}{2}$. Taking $t_{0}=\frac{1}{k} \log \left(\frac{3+\sqrt{5}}{2}\right)$, we have that the matrices $\rho_{k}\left(t_{0}\right)$ and $A$ are conjugate. In fact, put

$$
P=\left(\begin{array}{cccc}
1 & \frac{1}{2}(\sqrt{5}-1) & 0 & 0 \\
1 & -\frac{1}{2}(\sqrt{5}+1) & 0 & 0 \\
0 & 0 & 1 & \frac{1}{2}(\sqrt{5}-1) \\
0 & 0 & 1 & -\frac{1}{2}(\sqrt{5}+1)
\end{array}\right) .
$$

Then a direct calculation shows that $P A=\rho_{k}\left(t_{0}\right) P$. So, if $\left(m_{1}, m_{2}, m_{3}, m_{4}\right)^{t}$ is the transpose of the vector $\left(m_{1}, m_{2}, m_{3}, m_{4}\right)$, where $m_{1}, m_{2}, m_{3}, m_{4} \in \mathbb{Z}$, the lattice $\Gamma_{0}$ in $\mathbb{R}^{4}$ defined by

$$
\Gamma_{0}=P\left(m_{1}, m_{2}, m_{3}, m_{4}\right)^{t},
$$

is invariant under the subgroup $\mathbb{Z}$. Thus $\Gamma(k)=\left(t_{0} \mathbb{Z}\right) \ltimes_{\rho_{k}} \Gamma_{0}$ is a cocompact subgroup of $G(k)$. So, the quotient space

$$
S(k)=G(k) / \Gamma(k)
$$

is a 5-dimensional compact solvable manifold.

Alternatively, $S(k)$ may be viewed as the total space of a $T^{4}$-bundle over the circle $S^{1}$. In fact, let $T^{4}=\mathbb{R}^{4} / \Gamma_{0}$ be the 4-dimensional torus and $v: \mathbb{Z} \rightarrow \operatorname{Diff}\left(T^{4}\right)$ the representation defined as follows: $v(m)$ is the transformation of $T^{4}$ covered by the linear transformation of $\mathbb{R}^{4}$ given by the matrix

$$
\rho_{k}\left(m t_{0}\right)=\left(\begin{array}{cccc}
e^{k m t_{0}} & 0 & 0 & 0 \\
0 & e^{-k m t_{0}} & 0 & 0 \\
0 & 0 & e^{k m t_{0}} & 0 \\
0 & 0 & 0 & e^{-k m t_{0}}
\end{array}\right) .
$$

So $\mathbb{Z}$ acts on $T^{4} \times \mathbb{R}$ by 


$$
\left(\left(x_{1}, x_{2}, x_{3}, x_{4}\right), x_{5}\right) \mapsto\left(\rho_{k}\left(m t_{0}\right) \cdot\left(x_{1}, x_{2}, x_{3}, x_{4}\right)^{t}, x_{5}+m\right),
$$

and $S$ is the quotient $\left(T^{4} \times \mathbb{R}\right) / \mathbb{Z}$. The projection $\pi$ is given by

$$
\pi\left[\left(x_{1}, x_{2}, x_{3}, x_{4}\right), x_{5}\right]=\left[x_{5}\right] .
$$

Next, we consider the 7-dimensional compact manifold

$$
M^{7}(k)=S(k) \times T^{2},
$$

where $T^{2}$ is the 2-torus $T^{2}=\mathbb{R}^{2} / \mathbb{Z}^{2}$.

To compute the real cohomology of $M^{7}(k)$, we notice that $S(k)$ is completely solvable, that is the map $a d_{X}: \mathfrak{g}(k) \rightarrow \mathfrak{g}(k)$ has only real eigenvalues for all $X \in \mathfrak{g}(k)$, where $\mathfrak{g}(k)$ denotes the Lie algebra of $G(k)$. Thus Hattori's theorem [40] says that the de Rham cohomology ring $H^{*}(S(k))$ is isomorphic to the cohomology ring $H^{*}\left(\mathfrak{g}(k)^{*}\right)$ of the Lie algebra $\mathfrak{g}(k)$ of $G(k)$. For simplicity we denote the right invariant forms $\left\{e^{i}\right\}(i=1, \cdots, 5)$ on $G(k)$ and their projections on $S(k)$ by the same symbols. Then, if we denote by $e^{6}, e^{7}$ the (right invariant) closed 1-forms on the 2-torus $T^{2}$ whose cohomology classes generate the De Rham cohomology group $H^{1}\left(T^{2}, \mathbb{R}\right)$, we have that the 1 -forms $e^{i} \quad(1 \leq i \leq 7)$ on $M^{7}(k)$ are such that

$$
d e^{1}=-k e^{15}, \quad d e^{2}=k e^{25}, \quad d e^{3}=-k e^{35}, \quad d e^{4}=k e^{45}, \quad d e^{i}=0, i=5,6,7,
$$

and such that at each point of $M^{7}(k),\left\{e^{1}, e^{2}, e^{3}, e^{4}, e^{5}, e^{6}, e^{7}\right\}$ is a basis for the 1-forms on $M^{7}(k)$. Here $e^{15}$ stands for $e^{1} \wedge e^{5}$, and so on. Then, the real cohomology groups of $M^{7}(k)$ are:

$$
\begin{aligned}
& H^{0}\left(M^{7}(k)\right)=\langle 1\rangle, \\
& H^{1}\left(M^{7}(k)\right)=\left\langle\left[e^{5}\right],\left[e^{6}\right],\left[e^{7}\right]\right\rangle, \\
& H^{2}\left(M^{7}(k)\right)=\left\langle\left[e^{12}\right],\left[e^{14}\right],\left[e^{23}\right],\left[e^{34}\right],\left[e^{56}\right],\left[e^{57}\right],\left[e^{67}\right]\right\rangle, \\
& H^{3}\left(M^{7}(k)\right)=\left\langle\left[e^{125}\right],\left[e^{126}\right],\left[e^{127}\right],\left[e^{145}\right],\left[e^{146}\right],\left[e^{147}\right],\left[e^{235}\right],\right. \\
& {\left.\left[e^{236}\right],\left[e^{237}\right],\left[e^{345}\right],\left[e^{346}\right],\left[e^{347}\right],\left[e^{567}\right]\right\rangle, } \\
& H^{4}\left(M^{7}(k)\right)=\left\langle\left[e^{1234}\right],\left[e^{1256}\right],\left[e^{1257}\right],\left[e^{1267}\right],\left[e^{1456}\right],\left[e^{1457}\right],\left[e^{1467}\right],\right. \\
& {\left.\left[e^{2356}\right],\left[e^{2357}\right],\left[e^{2367}\right],\left[e^{3456}\right],\left[e^{3457}\right],\left[e^{3467}\right]\right\rangle, } \\
& H^{5}\left(M^{7}(k)\right)=\left\langle\left[e^{12345}\right],\left[e^{12346}\right],\left[e^{12347}\right],\left[e^{12567}\right],\left[e^{14567}\right],\left[e^{23567}\right],\left[e^{34567}\right]\right\rangle, \\
& H^{6}\left(M^{7}(k)\right)=\left\langle\left[e^{123456}\right],\left[e^{123457}\right],\left[e^{123467}\right]\right\rangle, \\
& H^{7}\left(M^{7}(k)\right)=\left\langle\left[e^{1234567}\right]\right\rangle .
\end{aligned}
$$

Thus, the Betti numbers of $M^{7}(k)$ are

$$
\begin{aligned}
& b_{0}\left(M^{7}(k)\right)=b_{7}\left(M^{7}(k)\right)=1, \\
& b_{1}\left(M^{7}(k)\right)=b_{6}\left(M^{6}(k)\right)=3, \\
& b_{2}\left(M^{7}(k)\right)=b_{5}\left(M^{7}(k)\right)=7, \\
& b_{3}\left(M^{7}(k)\right)=b_{4}\left(M^{7}(k)\right)=13 .
\end{aligned}
$$


Proposition 4.1. The 5-manifold $S(k)$ is 2-formal and so formal. Therefore, $M^{7}(k)=S(k) \times T^{2}$ is formal.

Proof. To prove that $S(k)$ is 2-formal, we see that its minimal model must be a differential graded algebra $(\mathcal{M}, d)$, where $\mathcal{M}$ is the free algebra of the form $\mathcal{M}=\Lambda\left(a_{1}\right) \otimes \Lambda\left(a_{2}, b_{2}, c_{2}, e_{2}\right) \otimes \Lambda V^{\geq 3}$, where the generator $a_{1}$ has degree 1 , the generators $a_{2}, b_{2}, c_{2}, e_{2}$ have degree 2 , and the differential $d$ is given by $d a_{1}=d a_{2}=d b_{2}=d c_{2}=d e_{2}=0$. The morphism $\rho: \mathcal{M} \rightarrow \Omega(S(k))$, inducing an isomorphism on cohomology, is defined by

$\rho\left(a_{1}\right)=e^{5}, \rho\left(a_{2}\right)=e^{12}, \rho\left(b_{2}\right)=e^{14}, \rho\left(c_{2}\right)=e^{23}$ and $\rho\left(e_{2}\right)=e^{34}$.

According to Definition 3.4, we get $C^{1}=\left\langle a_{1}\right\rangle$ and $N^{1}=0$, thus $S(k)$ is 1-formal. Moreover, $S(k)$ is 2-formal since $C^{2}=\left\langle a_{2}, b_{2}, c_{2}, e_{2}\right\rangle$ and $N^{2}=0$. Hence, $S(k)$ is 2 -formal, and so formal by Theorem 3.5. Now, Lemma 3.3 implies that $M^{7}(k)=S(k) \times T^{2}$ is formal.

We define the 3 -form $\varphi$ on $M^{7}(k)$ given by

$$
\varphi_{k}=e^{127}+e^{347}+e^{567}+e^{135}-e^{146}-e^{236}-e^{245} .
$$

Clearly, $\varphi_{k}$ is a $\mathrm{G}_{2}$ form on $M^{7}(k)$ which is closed. Indeed, on the right-hand side of (14) all the terms are closed, and so $\varphi_{k}$ is closed. Note that the dual form $\star_{\varphi_{k}} \varphi_{k}$ has the following expression

$$
\star_{\varphi_{k}} \varphi_{k}=e^{1234}+e^{1256}+e^{1367}+e^{1457}+e^{2357}-e^{2467}+e^{3456} .
$$

So, taking into account (11) and (12), we see that $e^{1367}$ and $e^{2467}$ are the unique nonclosed summands in $\star_{\varphi_{k}} \varphi_{k}$. In fact,

$d \star_{\varphi_{k}} \varphi_{k}=2 k\left(e^{13567}-e^{24567}\right) \neq 0$. Therefore, $\varphi_{k}$ does not define a torsion-free $\mathrm{G}_{2}$-structure on $M^{7}(k)$.

Now, let $H(k)$ be the simply connected solvable (non-nilpotent) Lie group $H(k)=G(k) \times \mathbb{R}^{2}$. Then, $\left\{e^{1}, e^{2}, e^{3}, e^{4}, e^{5}, e^{6}, e^{7}\right\}$ is a basis for the right invariant 1 -forms on $H(k)$ and the structure equations of $H(k)$ are given by (11). So, the closed $\mathrm{G}_{2}$ form $\varphi_{k}$ defined in (14) is a right invariant closed $\mathrm{G}_{2}$ form on $H(k)$.

Let $N$ be a simply connected solvable Lie group of dimension $n$, and denote by $\mathfrak{n}$ its Lie algebra. Recall that a right invariant metric $g$ on $\mathrm{N}$ is called a Ricci solsoliton metric (or simply solsoliton metric) if its Ricci endomorphism $\operatorname{Ric}(g)$ differs from a derivation $D$ of $\mathfrak{n}$ by a scalar multiple of the identity map $I_{n}$, i.e. if there exists a real number $\lambda$ such that

$$
\operatorname{Ric}(g)=\lambda I_{n}+D \text {. }
$$

Not all solvable Lie groups admit solsoliton metrics, but if a solsoliton exists, then it is unique up to automorphism and scaling [41].

Proposition 4.2. Let $H(k)$ be the seven dimensional Lie group $H(k)=G(k) \times \mathbb{R}^{2}$, and let $\varphi_{k}$ be the right invariant closed $\mathrm{G}_{2}$ form on $H(k)$ defined in (14). Then the metric $g_{k}$ determined by $\varphi_{k}$ is a solsoliton on $H(k)$.

Proof. Clearly, the metric $g_{k}$ induced on $H(k)$ by $\varphi_{k}$ is such that the 
basis $\left\{e^{1}, e^{2}, e^{3}, e^{4}, e^{5}, e^{6}, e^{7}\right\}$ for the 1-forms on $H(k)$ is orthonormal, that is $g_{k}=\sum_{i=1}^{7}\left(e^{i}\right)^{2}$. Then, $g_{k}$ is a solsoliton since

$$
\operatorname{Ric}\left(g_{k}\right)=\operatorname{diag}\left(0,0,0,0,-4 k^{2}, 0,0\right)=-4 k^{2} I_{7}+D,
$$

where

$$
D=\operatorname{diag}\left(4 k^{2}, 4 k^{2}, 4 k^{2}, 4 k^{2}, 0,4 k^{2}, 4 k^{2}\right),
$$

is a derivation of the Lie algebra $\mathfrak{h}(k)$ of $H(k)$.

\section{Associative 3-Folds in $M^{7}(k)$}

In this section, we show associative 3 -folds of the compact $\mathrm{G}_{2}$-calibrated solvmanifold $M^{7}(k)$ defined in (10) with the closed $\mathrm{G}_{2}$ form $\varphi_{k}$ given by (14). First, we need some definitions and results about calibrations (see [14] [15] for details).

Let $(M, g)$ be a Riemannian manifold. An oriented tangent $k$-plane $V$ on $M$ is a vector subspace $V$ of some tangent space $T_{p} M$ to $M$, with $\operatorname{dim} V=k$ and equipped with an orientation. If $V$ is an oriented tangent k-plane on $M$, then $g_{V}$ is a Euclidean metric on $V$. So, combining $g_{V}$ with the orientation on $V$ gives a natural volume form $\mathrm{vol}_{V}$ on $V$, which is a k-form on $V$.

Let $\theta$ a closed $k$-form on a Riemannian manifold $(M, g)$. We say that $\theta$ is a calibration on $M$ if for any $p \in M$ and every oriented $k$-dimensional subspace $V$ of the tangent space $T_{p} M$ we have $\left.\theta\right|_{V}=\lambda v o l_{V}$, for some $\lambda \leq 1$ (see [14] and [15] 3.7). Thus, if $Y$ is an oriented submanifold of $M$ with dimension $k$ then, for any $p \in Y$, the tangent space $T_{p} Y$ is an oriented tangent k-plane on $M$. We say that $Y$ is a calibrated submanifold if $\left.\theta(p)\right|_{T_{p} Y}=v l_{T_{p} Y}$, for all $p \in Y$.

All calibrated submanifolds are minimal submanifolds. Even more, every compact calibrated submanifold is volume-minimizing in its homology class ([15] Proposition 3.7.2).

Harvey and Lawson in [14] proved that any closed $\mathrm{G}_{2}$ form $\varphi$ on a 7-manifold $M$ is a calibration on $M$. The 3-dimensional orientable submanifolds $Y \subset M$ calibrated by the $\mathrm{G}_{2}$ form $\varphi$, i.e. those submanifolds $Y \subset M$ that satisfy $\left.\varphi(p)\right|_{T_{p} Y}=\operatorname{vol}_{Y}(p)$, for each $p \in Y$ and for some unique orientation of $Y$, are called associative 3 -folds.

Next, we shall produce examples of associative 3-folds in $M^{7}(k)$ from the fixed locus of a $\mathrm{G}_{2}$-involution of the compact manifold $M^{7}(k)$ applying the following.

Proposition 5.1 ([15] [Proposition 10.8.1]) Let $N$ be a 7-manifold with a closed $\mathrm{G}_{2}$ form $\phi$, and let $\sigma: N \rightarrow N$ be an involution of $N$ satisfying $\sigma^{*} \phi=\phi$ and such that $\sigma$ is not the identity map. Then the fixed point set $P=\{p \in N \mid \sigma(p)=p\}$ is an embedded associative 3-fold. Furthermore, if $N$ is compact then so is $P$.

Remark 5.2 Note that Proposition 10.8.1 in [15] is stated for the $\mathrm{G}_{2}$-structures 
that are closed and coclosed, but the coclosed condition is not used in the proof.

Proposition 5.3 There exist nine disjoint copies of 3-tori in $M^{7}(k)$, which define nine embedded, associative (calibrated by $\varphi_{k}$ ), minimal 3-tori in $M^{7}(k)$.

Proof. Let $H(k)$ be the seven dimensional Lie group $H(k)=G(k) \times \mathbb{R}^{2}$ defined in Proposition 4.2. We consider on $H(k)$ the involution given by

$$
\sigma:\left(x_{1}, x_{2}, x_{3}, x_{4}, x_{5}, x_{6}, x_{7}\right) \mapsto\left(-x_{1},-x_{2},-x_{3},-x_{4}, x_{5}, x_{6}, x_{7}\right),
$$

that is $\sigma$ is the product of the involutions $\sigma_{1}: G(k) \rightarrow G(k)$ with the identity map of $\mathbb{R}^{2}$, where $\sigma_{1}$ is defined by

$$
\sigma_{1}:\left(x_{1}, x_{2}, x_{3}, x_{4}, x_{5}\right) \mapsto\left(-x_{1},-x_{2},-x_{3},-x_{4}, x_{5}\right) .
$$

The involution $\sigma_{1}$ is such that $\sigma_{1}(\Gamma(k))=\Gamma(k)$, and so $\sigma_{1}$ descends to the 5-dimensional compact manifold $S(k)=G(k) / \Gamma(k)$. Hence, $\sigma$ defines also an involution of $M^{7}(k)$. From now on, we denote by

$$
\sigma: M^{7}(k) \rightarrow M^{7}(k)
$$

the involution of $M^{7}(k)$ induced by the involution $\sigma$ of $H(k)$ defined in (15). Then, taking into account (5), we have that the induced action on the 1 -forms $e^{i}$ is given by

$$
\sigma^{*} e^{i}=-e^{i}, i=1,2,3,4, \quad \rho^{*} e^{j}=e^{j}, j=5,6,7 .
$$

Therefore, the $\mathrm{G}_{2}$ form $\varphi_{k}$ on $M^{7}(k)$ defined in (14) is preserved by the involution $\sigma$ of $M^{7}(k)$. In fact, by (16), each term on the right-hand side of (14) is $\sigma$-invariant.

Let $P$ be the fixed locus of $\sigma$. Then, $P$ consists of all the 3-dimensional spaces $P_{a}$ given as follows:

$$
P_{a}=\left\{\left(a_{1}, a_{2}, a_{3}, a_{4}, x_{5}, x_{6}, x_{7}\right) \mid\left(x_{5}, x_{6}, x_{7}\right) \in T^{3}\right\} \subset M^{7}(k),
$$

where $\boldsymbol{a}=\left(a_{1}, a_{2}, a_{3}, a_{4}\right)$ with

$$
\left(a_{1}, a_{2}\right),\left(a_{3}, a_{4}\right) \in\left\{(0,0),\left(\frac{1}{2}, \frac{1}{2}\right), \frac{1}{4}(\sqrt{5}-1),-\frac{1}{4}(\sqrt{5}+1)\right\} .
$$

Consequently, $P$ is a disjoint union of 9 copies of a 3-torus $T^{3}$.

Since the $\mathrm{G}_{2}$ form $\varphi_{k}$ on $M^{7}(k)$ defined in (14) is preserved by the involution $\sigma$ of $M^{7}(k)$, each of the 9 torus $P_{a}$ in $M^{7}(k)$ fixed by $\sigma: M^{7}(k) \rightarrow M^{7}(k)$ is an associative 3-fold in $\left(M^{7}(k), \varphi_{k}\right)$ by Proposition 5.1 .

\section{The Laplacian Flow}

The purpose of this section is to prove that the Laplacian flow of $\varphi_{k}$ on the 7-dimensional Lie group $H(k)$ exists for all time. Moreover, we prove that the Ricci endomorphisms $\operatorname{Ric}\left(g_{k}(t)\right)$ of the underlying metrics $g_{k}(t)$ of the solution $\varphi_{k}(t)$ are independent of the time $t$, and so the solution $\varphi_{k}(t)$ does not converge to a torsion-free $\mathrm{G}_{2}$-structure as $t$ goes to infinity. 
Consider a 7-manifold $M$ endowed with a calibrated $\mathrm{G}_{2}$-structure $\varphi_{0}$. The Laplacian flow starting from $\varphi_{0}$ is the initial value problem

$$
\left\{\begin{array}{l}
\frac{d}{d t} \varphi(t)=\Delta_{t} \varphi(t), \\
d \varphi(t)=0, \\
\varphi(0)=\varphi_{0} .
\end{array}\right.
$$

where $\Delta_{t}$ denotes the Hodge Laplacian of the Riemannian metric $g(t)$ induced by $\varphi(t)$. This flow was introduced by Bryant in [16] to study seven-dimensional manifolds admitting calibrated $\mathrm{G}_{2}$-structures. Notice that the stationary points of the flow Equation in (17) are harmonic $\mathrm{G}_{2}$-structures, which coincide with torsion-free $\mathrm{G}_{2}$-structures on compact manifolds.

Short-time existence and uniqueness of the solution of (17) when $M$ is compact were proved in [20].

Theorem 6.1 Assume that $M$ is compact. Then, the Laplacian flow (17) has a unique solution defined for a short time $t \in[0, \varepsilon)$, with $\varepsilon$ depending on $\varphi_{0}$.

In the following theorem, we determine a global solution of the Laplacian flow of the closed $\mathrm{G}_{2}$ form $\varphi_{k}$ given by (14) on the Lie group $H(k)=G(k) \times \mathbb{R}^{2}$, where $G(k)$ is the Lie group defined in Section 4 .

Theorem 6.2 On the simply connected solvable (non-nilpotent) Lie group $H(k)=G(k) \times \mathbb{R}^{2}$, the solution of the Laplacian flow (17) starting from the calibrated $\mathrm{G}_{2}$-structure $\varphi_{k}$ is given by

$$
\varphi_{k}(t)=e^{127}+e^{347}+e^{567}+\left(\frac{16}{3} k t+1\right)^{3 / 4} e^{135}-e^{236}-e^{146}-\left(\frac{16}{3} k t+1\right)^{3 / 4} e^{245},
$$

where $t \in\left(-\frac{3}{16 k^{2}},+\infty\right)$

Proof. Let $f_{i}=f_{i}(t) \quad(i=1, \cdots, 7)$ be some differentiable real functions depending on a parameter $t \in I \subset \mathbb{R}$ such that $f_{i}(0)=1$ and $f_{i}(t) \neq 0$, for any $t \in I$, where $I$ is a real open interval. For each $t \in I$, we consider the basis $\left\{x^{1}, \cdots, x^{7}\right\}$ of left invariant 1 -forms on $H(k)$ defined by

$$
x^{i}=x^{i}(t)=f_{i}(t) e^{i}, \quad 1 \leq i \leq 7 .
$$

Taking into account (11), the structure equations of $H(k)$ with respect to the basis $\left\{x^{1}, \cdots, x^{7}\right\}$ are

$$
\begin{aligned}
& d x^{1}=-k \frac{1}{f_{5}} x^{15}, \quad d x^{2}=k \frac{1}{f_{5}} x^{25}, \\
& d x^{3}=-k \frac{1}{f_{5}} x^{35}, \quad d x^{4}=k \frac{1}{f_{5}} x^{45}, \\
& d x^{5}=d x^{6}=d x^{7}=0 .
\end{aligned}
$$

From now on, we write $f_{i j}=f_{i j}(t)=f_{i}(t) f_{j}(t)$, $f_{i j k}=f_{i j k}(t)=f_{i}(t) f_{j}(t) f_{k}(t)$, and so forth. Then, for any $t \in I$, we consider the $\mathrm{G}_{2}$-structure $\varphi_{k}(t)$ on $H(k)$ given by 


$$
\begin{aligned}
\varphi_{k}(t) & =x^{127}+x^{347}+x^{567}+x^{135}-x^{146}-x^{236}-x^{245} \\
& =f_{127} e^{127}+f_{347} e^{347}+f_{567} e^{567}+f_{135} e^{135}-f_{146} e^{146}-f_{236} e^{236}-f_{245} e^{245} .
\end{aligned}
$$

Note that the 3 -form $\varphi_{k}(t)$ defined by (20) is such that $\varphi_{k}(0)=\varphi_{k}$ and, for any $t, \varphi_{k}(t)$ determines the metric $g_{k}(t)$ on $H(k)$ such that the basis $\left\{x_{i}=\frac{1}{f_{i}} e_{i} ; i=1, \cdots, 7\right\}$ of left invariant vector fields on $H(k)$ dual to $\left\{x^{1}, \cdots, x^{7}\right\}$ is orthonormal. Moreover, by (19), $\varphi_{k}(t)$ is closed, for any $t \in I$. Therefore, to solve the flow (17) of $\varphi_{k}$ it is sufficient to determine the functions $f_{i}$ and the interval $I$ so that $\frac{d}{d t} \varphi_{k}(t)=\Delta_{t} \varphi_{k}(t)$, for $t \in I$.

Clearly $\Delta_{t} \varphi_{k}(t)=-d \star_{t} d \star_{t} \varphi_{k}(t)$ since $d \varphi_{k}(t)=0$. Moreover,

$$
\star_{t} \varphi_{k}(t)=x^{1234}+x^{1256}+x^{1367}+x^{1457}+x^{2357}-x^{2467}+x^{3456} .
$$

So, $x^{1367}$ and $x^{2467}$ are the unique nonclosed summands in $\star_{t} \varphi_{k}(t)$. Then, taking into account (19), we obtain

$$
\Delta_{t} \varphi_{k}(t)=\frac{4 k^{2}}{f_{5}^{2}}\left(x^{135}-x^{245}\right) .
$$

Thus, in terms of the forms $e^{i j k}$, the expression of $\Delta_{t} \varphi_{k}(t)$ becomes

$$
\Delta_{t} \varphi_{k}(t)=\frac{4 k^{2}}{f_{5}}\left(f_{13} e^{135}-f_{24} e^{245}\right) .
$$

On the other hand,

$$
\begin{aligned}
\frac{d}{d t} \varphi_{k}(t)= & \left(f_{127}\right)^{\prime} e^{127}+\left(f_{347}\right)^{\prime} e^{347}+\left(f_{567}\right)^{\prime} e^{567}+\left(f_{135}\right)^{\prime} e^{135} \\
& -\left(f_{146}\right)^{\prime} e^{146}-\left(f_{236}\right)^{\prime} e^{236}-\left(f_{245}\right)^{\prime} e^{245}
\end{aligned}
$$

Comparing (21) and (22) we have that $\frac{d}{d t} \varphi_{k}(t)=\Delta_{t} \varphi_{k}(t)$ if and only if the functions $f_{i}$ satisfy the following equations

$$
\begin{gathered}
\left(f_{127}\right)^{\prime}=\left(f_{347}\right)^{\prime}=\left(f_{567}\right)^{\prime}=\left(f_{236}\right)^{\prime}=\left(f_{146}\right)^{\prime}=0, \\
\left(f_{135}\right)^{\prime}=4 k^{2} \frac{f_{13}}{f_{5}}, \\
\left(f_{245}\right)^{\prime}=4 k^{2} \frac{f_{24}}{f_{5}} .
\end{gathered}
$$

The equations (23) with the initial conditions $f_{i}(0)=1 \quad(i=1, \cdots, 7)$ imply $f_{127}=f_{347}=f_{567}=f_{236}=f_{146}=1$.

Now, the equalities $f_{127}=f_{347}$ and $f_{236}=f_{146}$ imply $f_{12}=f_{34}$ and $f_{23}=f_{14}$, respectively, and thus

$$
f_{1}=f_{3} \text { and } f_{2}=f_{4} .
$$

Moreover, from $f_{127}=f_{236}=1$ we have

$$
f_{6}=f_{7}=1 / f_{12},
$$


and from $f_{567}=1$ we have

$$
f_{5}=f_{12}^{2}
$$

Now, using (26) and (28), the system of differential equations formed by the Equations (24) and (25) is written as

$$
\left\{\begin{array}{l}
\left(f_{1}^{2}\left(f_{1} f_{2}\right)^{2}\right)^{\prime}=4 k^{2} \frac{1}{f_{2}^{2}} \\
\left(f_{2}^{2}\left(f_{1} f_{2}\right)^{2}\right)^{\prime}=4 k^{2} \frac{1}{f_{1}^{2}} .
\end{array}\right.
$$

Multiplying the first equation of (29) by $f_{2}^{2}$, and the second one by $f_{1}^{2}$, one can check that (29) implies that

$$
f_{2}^{2}\left(f_{1}^{4} f_{2}^{2}\right)^{\prime}=f_{1}^{2}\left(f_{1}^{2} f_{2}^{4}\right)^{\prime},
$$

that is,

$$
f_{2}\left(f_{1}\right)^{\prime}=f_{1}\left(f_{2}\right)^{\prime} .
$$

Then, using that $f_{1}(0)=f_{2}(0)=1$, we have

$$
f_{1}=f_{2} \text {. }
$$

Thus, the system (29) is written as follows

$$
\left(f_{1}^{6}\right)^{\prime}=4 k^{2} \frac{1}{f_{1}^{2}}
$$

Integrating this equation, we obtain

$$
\frac{3}{4} f_{1}^{8}=4 k^{2} t+C
$$

for some constant $C \in \mathbb{R}$. But the initial condition $f_{1}(0)=1$ implies $C=\frac{3}{4}$, and hence

$$
f_{1}(t)=\sqrt[8]{\frac{16}{3} k^{2} t+1}
$$

From (26), (27), (28), (30) and (31), we get

$$
\begin{aligned}
& f_{1}(t)=f_{2}(t)=f_{3}(t)=f_{4}(t)=\sqrt[8]{\frac{16}{3} k^{2} t+1} \\
& f_{5}(t)=\sqrt{\frac{16}{3} k^{2} t+1}, \quad f_{6}(t)=f_{7}(t)=\frac{1}{\sqrt[4]{\frac{16}{3} k^{2} t+1}} .
\end{aligned}
$$

Therefore, taking into account (20), the family of closed $\mathrm{G}_{2}$ forms $\varphi_{k}(t)$ given by (18) is the solution of the Laplacian flow of $\varphi_{k}$ on $H(k)$, and it is defined for all $t \in\left(-\frac{3}{16 k^{2}},+\infty\right)$.

Remark 6.3 Note that the metric $g_{k}(t)$, with $t \in\left(-\frac{3}{16 k^{2}},+\infty\right)$, is a 
solsoliton on $H(k)$. In fact, the metric $g_{k}(t)$ with respect to the basis $\left\{e_{1}, \cdots, e_{7}\right\}$ is given by

$$
g_{k}(t)=\operatorname{diag}\left(f_{1}^{2}, f_{1}^{2}, f_{1}^{2}, f_{1}^{2}, f_{1}^{8}, \frac{1}{f_{1}^{4}}, \frac{1}{f_{1}^{4}}\right),
$$

where $f_{1}=f_{1}(t)$ is the function given by (31). Then, the Ricci endomorphism $\operatorname{Ric}\left(g_{k}(t)\right)$ satifies

$$
\operatorname{Ric}\left(g_{k}(t)\right)=\operatorname{diag}\left(0,0,0,0,-4 k^{2}, 0,0\right)=-4 k^{2} I_{7}+D,
$$

where

$$
D=\operatorname{diag}\left(4 k^{2}, 4 k^{2}, 4 k^{2}, 4 k^{2}, 0,4 k^{2}, 4 k^{2}\right),
$$

is a derivation of the Lie algebra $\mathfrak{h}(k)$ of $H(k)$. Moreover, $\operatorname{Ric}\left(g_{k}(t)\right)$ on $H(k)$ is non-zero and independent of the time $t$. So, the solution $\varphi_{k}(t)$ does not converge to a torsion-free $\mathrm{G}_{2}$-structure as $t$ goes to infinity.

Furthermore, taking into account the symmetry properties of the Riemannian curvature $R\left(g_{k}(t)\right)$ we obtain

$$
\begin{aligned}
& R_{1212}=R_{1414}=R_{2323}=R_{3434}=\frac{k^{2}}{f_{1}^{4}}, \\
& R_{1313}=R_{2424}=-\frac{k^{2}}{f_{1}^{4}}, \\
& R_{1515}=R_{2525}=R_{3535}=R_{4545}=-k^{2} f_{1}^{2}, \\
& R_{i j k l}=0 \text { otherwise, }
\end{aligned}
$$

where $R_{i j k l}=R\left(g_{k}(t)\right)\left(e_{i}, e_{j}, e_{k}, e_{l}\right)$. Thus, the Riemannian curvature $R\left(g_{k}(t)\right)$ does not converge when $t$ tends to infinity.

\section{Acknowledgements}

The authors were partially supported by MINECO-FEDER Grant MTM2014-54804-P and Gobierno Vasco Grant IT1094-16, Spain.

\section{Conflicts of Interest}

The authors declare no conflicts of interest regarding the publication of this paper.

\section{References}

[1] Fernández, M. and Gray, A. (1982) Riemannian Manifolds with Structure Group G. Annali di Matematica Pura ed Applicata, 132, 19-45. https://doi.org/10.1007/BF01760975

[2] Salamon, S. (1989) Riemannian Geometry and Holonomy Groups. Longman Scientific and Technical, Harlow Essex, UK.

[3] Joyce, D.D. (1996) Compact Riemannian 7-Manifolds with Holonomy G2. I, II. Journal of Differential Geometry, 43, 291-328, 329-375.

[4] Kovalev, A. (2003) Twisted Connected Sums and Special Riemannian Holonomy. Journal für die Reine und Angewandte Mathematik, 565, 125-160. 
https://doi.org/10.1515/crll.2003.097

[5] Corti, A., Haskins, M., Nordström, J. and Pacini, T. (2015) G ${ }_{2}$-Manifolds and Associative Submanifolds via Semi-Fano 3-Folds. Duke Mathematical Journal, 164, 1971-2092. https://doi.org/10.1215/00127094-3120743

[6] Joyce, D.D. and Karigiannis, S. A New Construction of Compact Torsion-Free $\mathrm{G}_{2}$-Manifolds by Gluing Families of Eguchi-Hanson Spaces. To Appear in Journal of Differential Geometry. arXiv:1707.09325 [math.DG].

[7] Bryant, R.L. (1987) Metrics with Exceptional Holonomy. Annals of Mathematics, 126, 525-576.

[8] Fernández, M. (1987) An Example of a Compact Calibrated Manifold Associated with the Exceptional Lie Group $\mathrm{G}_{2}$. Journal of Differential Geometry, 26, 367-370. https://doi.org/10.4310/jdg/1214441377

[9] Conti, D. and Fernández, M. (2011) Nilmanifolds with a Calibrated $\mathrm{G}_{2}$-Structure. Differential Geometry and its Applications, 29, 493-506. https://doi.org/10.1016/j.difgeo.2011.04.030

[10] Fernández, M. (1987) A Family of Compact Solvable G2-Calibrated Manifolds. Tohoku Mathematical Journal, 30, 287-289. https://doi.org/10.2748/tmj/1178228331

[11] Fernández, M., Fino, A., Kovalev, A. and Muñoz, V. A Compact $\mathrm{G}_{2}$-Calibrated Manifold with $b_{1}=1$. arXiv:1808.07144 [math.DG].

[12] Fernández, M., Manero, V., Otal, A. and Ugarte, L. (2013) Symplectic Half-Flat Solvmanifolds. Annals of Global Analysis and Geometry, 43, 367-383. https://doi.org/10.1007/s10455-012-9349-6

[13] Fino, A. and Raffero, A. Closed $\mathrm{G}_{2}$-Structures on Non-Solvable Lie Groups. arXiv:1712.09664 [math.DG].

[14] Harvey, R. and Lawson Jr., H.B. (1982) Calibrated Geometries. Acta Mathematica, 148, 47-157. https://doi.org/10.1007/BF02392726

[15] Joyce, D.D. (2008) Compact Manifolds with Special Holonomy. OSP, Oxford.

[16] Bryant, R.L. (2006) Some Remarks on $\mathrm{G}_{2}$-Structures. Proceedings of Gökova Geometry- Topology Conference, Gökova, 75-109.

[17] Cleyton, R. and Ivanov, S. (2007) On the Geometry of Closed $\mathrm{G}_{2}$-Structures. Communications in Mathematical Physics, 270, 53-67. https://doi.org/10.1007/s00220-006-0145-7

[18] Fernández, M., Fino, A. and Manero, V. (2015) G2-Structures on Einstein Solvmanifolds. Asian Journal of Mathematics, 19, 321-342. https://doi.org/10.4310/AJM.2015.v19.n2.a7

[19] Hamilton, R.S. (1988) The Ricci Flow on Surfaces. Contemporary Mathematics, 71, 237-261. https://doi.org/10.1090/conm/071/954419

[20] Bryant, R.L. and Xu, F. (2004) Laplacian Flow for Closed G2-Structures: Short Time Behavior. arXiv:1101.2004 [math.DG]

[21] Lotay, J.D. and Wei, Y. (2017) Laplacian Flow for Closed $G_{2}$ Structures: Shi-Type Estimates, Uniqueness and Compactness. Geometric and Functional Analysis, 27, 165-233. https://doi.org/10.1007/s00039-017-0395-X

[22] Lotay, J.D. and Wei, Y. (2015) Stability of Torsion-Free $G_{2}$ Structures along the Laplacian Flow. arXiv:1504.07771 [math.DG]

[23] Lotay, J.D. and Wei, Y. (2016) Laplacian Flow for Closed G 2 Structures: Real Analyticity. arXiv:1601.04258 [math.DG] 
[24] Fernández, M., Fino, A. and Manero, V. (2016) Laplacian Flow of Closed $\mathrm{G}_{2}$-Structures Inducing Nilsolitons. Journal of Geometric Analysis, 26, 1808-1837. https://doi.org/10.1007/s12220-015-9609-3

[25] Fino, A. and Raffero, A. (2018) Closed Warped $\mathrm{G}_{2}$-Structures Evolving under the Laplacian Flow. arXiv:1708.00222 [math.DG]

[26] Lauret, J. (2017) Laplacian Flow of Homogeneous $\mathrm{G}_{2}$-Structures and Its Solitons. Proceedings of the London Mathematical Society, 114, 527-560. https://doi.org/10.1112/plms.12014

[27] Lauret, J. (2017) Laplacian Solitons: Questions and Homogeneous Examples. Differential Geometry and Its Applications, 54, 345-360.

[28] Nicolini, M. (2016) Laplacian Solitons on Nilpotent Lie Groups. arXiv:1608.08599 [math.DG]

[29] Huang, H., Wang, Y. and Yao, C. (2017) Cohomogeneity-One G2-Laplacian Flow on 7-Torus. arXiv:1709.02149 [math.DG]

[30] Bryant, R.L. (2010) Non-Embedding and Non-Extension Results in Special Holonomy. In: The Many Facets of Geometry, Oxford Univ. Press, Oxford, 346-367. https://doi.org/10.1093/acprof:oso/9780199534920.003.0017

[31] Donaldson, S. (2018) Some Recent Developments in Kähler Geometry and Exceptional Holonomy. arXiv: 1808.03995 [math.DG]

[32] Hitchin, N. (2000) The Geometry of Three-Forms in Six and Seven Dimensions. Journal of Differential Geometry, 55, 547-576. https://doi.org/10.4310/jdg/1090341263

[33] Hitchin, N. (2001) Stable Forms and Special Metrics. In: Fernandez, M. and Wolf, J., Eds., Global Differential Geometry: The Mathematical Legacy of Alfred Gray, Vol. 288, American Mathematical Society, Providence, 70-89.

[34] Gray, A. (1969) Vector Cross Products on Manifolds. Transactions of the American Mathematical Society, 141, 463-504. https://doi.org/10.1090/S0002-9947-1969-0243469-5

[35] Bonan, E. (1966) Sur des variétés riemanniennes à groupe d'holonomie $\mathrm{G}_{2}$ ou Spin (7). Comptes Rendus de P Académie des Sciences A-B, 262, A127-A129.

[36] Halperin, S. (1983) Lectures on Minimal Models.

[37] Deligne, P., Griffiths, P., Morgan, J. and Sullivan, D. (1975) Real Homotopy Theory of Kähler Manifolds. Inventiones Mathematicae, 29, 245-274. https://doi.org/10.1007/BF01389853

[38] Griffiths, P.A. and Morgan, J.W. (1981) Rational Homotopy Theory and Differential Forms. Progress in Mathematics, Vol. 16, Birkhäuser, Boston.

[39] Fernández, M. and Muñoz, V. (2005) Formality of Donaldson Submanifolds. Mathematische Zeitschrift, 250, 149-175. https://doi.org/10.1007/s00209-004-0747-8

[40] Hattori, A. (1960) Spectral Sequences in the de Rham Cohomology of Fibre Bundles. Journal of the Faculty of Science, University of Tokyo. Section I, 8, 289-331.

[41] Lauret, J. (2001) Ricci Soliton Homogeneous Nilmanifolds. Mathematische Annalen, 319, 715-733. https://doi.org/10.1007/PL00004456 